\title{
Bax targets mitochondria by distinct mechanisms before or during apoptotic cell death: a requirement for VDAC2 or Bak for efficient Bax apoptotic function
}

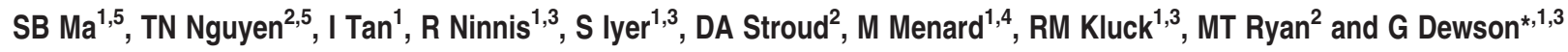

In non-apoptotic cells, Bak constitutively resides in the mitochondrial outer membrane. In contrast, Bax is in a dynamic equilibrium between the cytosol and mitochondria, and is commonly predominant in the cytosol. In response to an apoptotic stimulus, Bax and Bak change conformation, leading to Bax accumulation at mitochondria and Bak/Bax oligomerization to form a pore in the mitochondrial outer membrane that is responsible for cell death. Using blue native-PAGE to investigate how Bax oligomerizes in the mitochondrial outer membrane, we observed that, like Bak, a proportion of Bax that constitutively resides at mitochondria associates with voltage-dependent anion channel (VDAC)2 prior to an apoptotic stimulus. During apoptosis, Bax dissociates from VDAC2 and homo-oligomerizes to form high molecular weight oligomers. In cells that lack VDAC2, constitutive mitochondrial localization of Bax and Bak was impaired, suggesting that VDAC2 has a role in Bax and Bak import to, or stability at, the mitochondrial outer membrane. However, following an apoptotic stimulus, Bak and Bax retained the ability to accumulate at VDAC2-deficient mitochondria and to mediate cell death. Silencing of Bak in VDAC2-deficient cells indicated that Bax required either VDAC2 or Bak in order to translocate to and oligomerize at the mitochondrial outer membrane to efficiently mediate apoptosis. In contrast, efficient Bak homo-oligomerization at the mitochondrial outer membrane and its pro-apoptotic function required neither VDAC2 nor Bax. Even a C-terminal mutant of Bax (S184L) that localizes to mitochondria did not constitutively target mitochondria deficient in VDAC2, but was recruited to mitochondria following an apoptotic stimulus dependent on Bak or upon over-expression of $\mathrm{Bcl}-\mathrm{x}_{\mathrm{L}}$. Together, our data suggest that Bax localizes to the mitochondrial outer membrane via alternate mechanisms, either constitutively via an interaction with VDAC2 or after activation via interaction with Bcl-2 family proteins. Cell Death and Differentiation (2014) 21, 1925-1935; doi:10.1038/cdd.2014.119; published online 22 August 2014

Bax and Bak are the key effectors of the intrinsic apoptotic pathway initiated in response to diverse stimuli including anoikis, DNA damage and growth factor withdrawal. ${ }^{1}$ Both proteins are normally dormant in healthy cells, but upon reception of an apoptotic stimulus, they undergo conformation change that allows their self-association to form pores in the mitochondrial outer membrane (MOM) ${ }^{2-7}$ The consequence of disruption of the MOM is twofold; it impairs the ability of mitochondria to generate ATP by oxidative phosphorylation and it allows the release of intermembrane proteins including cytochrome $c$ that agonizes caspases that dismantle the cell.

Bak and Bax share significant structural homology in their inactive states and have conserved mechanism of conformation change and oligomerization. ${ }^{3,8-10}$ Further, genetic studies reveal that Bak and Bax perform at least partially overlapping function, with deficiency in both necessary to perturb apoptosis during embryonic development and in response to toxic insult. ${ }^{1,11}$ However, whether Bak and Bax are regulated similarly is unclear. Whereas Bak is constitutively anchored in the MOM via its hydrophobic C-terminal transmembrane domain, Bax is predominantly cytosolic in the majority of non-apoptotic cells. ${ }^{12}$ Recent evidence indicates that Bax is in a dynamic equilibrium between cytosol and mitochondria and is constantly trafficked away from the MOM in non-apoptotic cells. ${ }^{13,14}$ In response to apoptotic stress this 'retrotranslocation' is disrupted causing Bax to accumulate at mitochondria; a hallmark of most apoptotic cells. The mechanism governing the dynamic distribution of Bax in healthy and apoptotic cells is unclear with interactions with pro-survival proteins debated. ${ }^{13,14}$

Voltage-dependent anion channels (VDACs) are the major channels responsible for ion passage across the MOM. Studies have also implicated an additional role for the VDACs in the regulation of Bak or Bax apoptotic function or potentially even constituting a component of the Bak/Bax apoptotic pore. ${ }^{15-18}$ However, these studies have provided contrasting

\footnotetext{
${ }^{1}$ Walter and Eliza Hall Institute of Medical Research, $1 \mathrm{G}$ Royal Parade, Parkville, Melbourne, Victoria, Australia; ${ }^{2}$ Department of Biochemistry, La Trobe Institute for Molecular Science, La Trobe University, Melbourne, Australia; ${ }^{3}$ Department of Medical Biology, University of Melbourne, Parkville, Victoria and ${ }^{4}$ Centre de Cancérologie de Lyon, Université de Lyon, Lyon, France

${ }^{*}$ Corresponding author: G Dewson, Walter and Eliza Hall Institute of Medical Research, 1 G Royal Parade, Parkville, Victoria 3052, Australia. Tel: +61 393452935 ; Fax: +61 3 93470852; E-mail: dewson@ @ehi.edu.au

${ }^{5}$ These authors contributed equally to this work.

Abbreviations: BH, Bcl-2 homology; BN-PAGE, blue native-polyacrylamide gel electrophoresis; C-terminus, carboxy terminus; DTT, dithiothreitol; GFP, green fluorescent protein; HA, haemagglutinin; hBax, human Bax; IRES, internal ribosome entry site; mBax, mouse Bax; MEFs, mouse embryonic fibroblasts; MOM, mitochondrial outer membrane; N-terminus, amino terminus; PI, propidium iodide; SDS-PAGE, sodium dodecyl sulphate-polyacrylamide gel electrophoresis; sh, short hairpin; TALEN, transcription activator-like effector nuclease; tBid, truncated Bid; VDAC, voltage-dependent anion channel

Received 01.1.14; revised 24.6.14; accepted 13.7.14; Edited by C Borner; published online 22.8.14
} 
findings relating to whether VDACs might positively or negatively regulate Bak/Bax apoptotic function.

We used blue native-PAGE (BN-PAGE) to investigate how Bax oligomerizes in the MOM during apoptosis. We observed that VDAC2 is a determinant of the constitutive association of both Bax and Bak with the MOM. The defect in Bax mitochondrial localization can be bypassed by Bak-dependent recruitment during apoptosis. Thus, our data suggest that mitochondrial localization of Bax occurs via distinct mechanisms in healthy and apoptotic cells and that either VDAC2 or Bak is required for the efficient translocation of Bax and hence for the oligomerization at the MOM and Bax apoptotic function.

\section{Results}

Bax associates with a discrete high molecular weight complex requiring VDAC2 in non-apoptotic cells. In healthy mouse embryonic fibroblasts (MEFs), Bax is predominantly cytosolic, but with a population that constitutively resides at mitochondria (Figure 1a). ${ }^{12,14} \mathrm{Bak}$, on the other hand, is constitutively mitochondrial (Figure 1a). To examine the oligomeric state of Bax at each subcellular location, we analysed $\mathrm{Bak}^{-/-}$MEFs or $\mathrm{Bak}^{-/-} \mathrm{Bax}^{-/-}$MEFs stably expressing human Bax by BN-PAGE. To analyse Bax complexes, we used digitonin as it retains Bax in its native conformation $^{5}$ and retains protein-protein interactions of $\mathrm{Bax}^{19}$ and Bak. ${ }^{20,21}$ Although the zwitterionic detergent CHAPS is thought to maintain Bax in its inactive state, ${ }^{5,12}$ it has also been reported to disrupt Bax interactions, ${ }^{19}$ and fails to efficiently solubilize certain MOM proteins such as VDAC $1 .{ }^{22}$ When solubilized in digitonin, but not CHAPS or Triton X-100, distinct Bax complexes in subcellular fractions (cytosol and mitochondria-enriched heavy membrane) of non-apoptotic or apoptotic cells were observed (Figure 1b). Endogenous Bax in $\mathrm{Bak}^{-/-}$MEFs formed similar complexes to ectopically expressed hBax (Figure 1c) and none were detected in $\mathrm{Bak}^{-/-} \mathrm{Bax}^{-/-}$MEFs (Figure 1d) thereby confirming specificity.

Prior to an apoptotic stimulus, Bax in the cytosol and mitochondria-enriched membrane fractions was detected in a similar low molecular weight form (Figures $1 \mathrm{~b}$ and $\mathrm{c}$ ). Bax has been previously shown to be monomeric in the cytosol of healthy cells, ${ }^{12,23}$ suggesting that the mitochondrial population was likewise monomeric. However, a population of Bax was additionally resident in a high molecular weight ( $>400 \mathrm{kDa}$ ) complex in mitochondria of MEFs and a variety of cells, including primary and of different genetic backgrounds (Supplementary Figure 2). We confirmed that the complex was not an artefact of disulphide-linkage during solubilization as a Bax mutant that lacks both endogenous cysteines similarly localized to this complex (Figure 1e). A concentration of digitonin $(1 \% \mathrm{w} / \mathrm{v}, 8 \mathrm{mM})$ significantly above its critical micelle concentration $(0.5 \mathrm{mM})$ was required to efficiently solubilize the high molecular weight complex of Bax (Figure 1f) and likewise the membrane-integrated MOM protein VDAC1 (Supplementary Figure 2). In contrast, monomeric Bax could be solubilized from the MOM at a low concentration of digitonin $(<0.1 \% \mathrm{w} / \mathrm{v}, 0.8 \mathrm{mM})$, consistent with monomeric Bax being peripherally attached to the MOM in healthy cells (Supplementary Figure 2). Increasing digitonin concentrations did not disrupt the high molecular weight form of Bax, but instead slightly retarded the migration of monomeric Bax on BN-PAGE (Figure $1 f$ and Supplementary Figure 3, compare lanes 3 and 7 ), consistent with a previous report for other membrane-associated proteins. ${ }^{22}$

The localization of Bax to a high molecular weight complex in mitochondria of non-apoptotic MEFs was reminiscent of Bak complexing with VDAC2. ${ }^{20,21}$ To assess whether Bax also associates with VDAC2, we analysed $\mathrm{Vdac}^{-/-}$MEFs. As with Bak, ${ }^{20,21}$ the high molecular weight Bax complex was undetectable in $\mathrm{Vdac2}^{-/-}$MEFs (Figure 2a) and also HCT116 cells where VDAC2 was disrupted using TALEN ( $\triangle V D A C 2$, Figure $2 \mathrm{~b}$ and Supplementary Figure 3 ). The VDAC2-Bax complex was not observed when VDAC2proficient mitochondria were solubilized in the presence of cytosolic Bax confirming that the complex was not driven by digitonin (Supplementary Figure 4). Ectopic expression of a haemagglutinin (HA)-tagged VDAC2 in $\mathrm{Vdac2}^{-/-}$MEFs restored the large molecular weight Bax complex and also monomeric Bax in the MOM in healthy cells (Figure 2c, compare lanes 2 and 6 and Supplementary Figure 5). Antibody gel-shift assay confirmed that Bax and VDAC2 reside in the same complex (Supplementary Figure 6). Thus, endogenous Bax (Figure 2a), as well as Bak, ${ }^{15,20}$ associates with a large molecular weight complex involving VDAC2 in mitochondria of healthy cells. This contrasts with other Bcl-2 family proteins, for example, neither $\mathrm{Bcl}-\mathrm{x}_{\mathrm{L}}$ nor $\mathrm{Bcl}-2$ associated with this large complex when imported into mitochondria (Supplementary Figure 7).

Bax dissociates from VDAC2 and appears in a range of oligomeric complexes during apoptosis. Upon reception of an apoptotic stimulus (etoposide, staurosporine or actinomycin D) a ladder of Bax complexes from 60 to $400 \mathrm{kDa}$ was detected (Figures 1d,e and 2a, Supplementary Figure 8$).{ }^{5}$ Although changes in oligomerization state during apoptosis were observed in CHAPS, this detergent did not resolve discrete complexes (Figure 1b), consistent with a previous report. ${ }^{24}$ In apoptotic cells, Bax oligomers could only be extracted with $>0.25 \%$ digitonin, consistent with activated Bax being membrane-integrated. As in healthy cells, monomeric Bax in apoptotic cells was peripheral as it could be extracted when membranes were resuspended in low concentrations of digitonin $(0.1 \% \mathrm{w} / \mathrm{v})$ (Figure $1 \mathrm{f})$ or even in the absence of digitonin (Supplementary Figure 9). This suggests that during apoptosis, Bax integration and oligomerization occur concurrently, or that Bax integration precedes oligomerization as suggested by previous studies. ${ }^{24,25}$

The apoptotic Bax oligomers were not stabilized by disulphide linkage as they were similarly detected with a Bax $\Delta$ Cys variant (Figure 1e). The pattern of oligomeric Bax contrasts with oligomeric Bak, which under native (and reducing) conditions resolves predominantly as a homodimer. ${ }^{21}$ This may suggest that Bax and Bak high molecular weight oligomeric pores form by different mechanisms. However, as Bax and Bak can associate to form higher order oligomers, because of their conserved mechanism of symmetrical homodimerization, distinct mechanisms of pore formation appear unlikely. ${ }^{5,21,26,27}$ A more likely explanation is that 
a

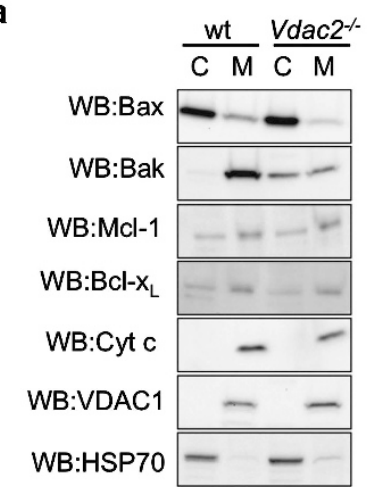

b

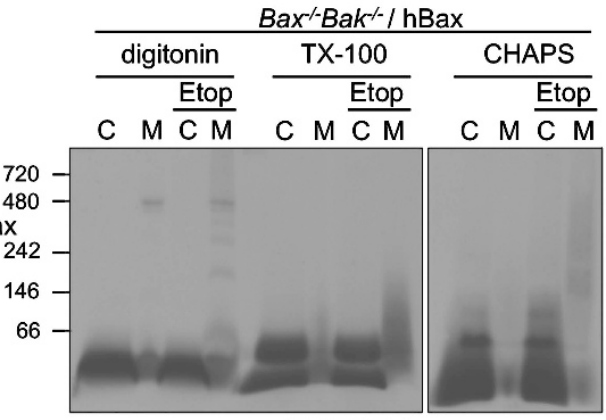

BN-PAGE

d

c

Bax ${ }^{-1}$ Bak $^{\prime-1}$ hBax

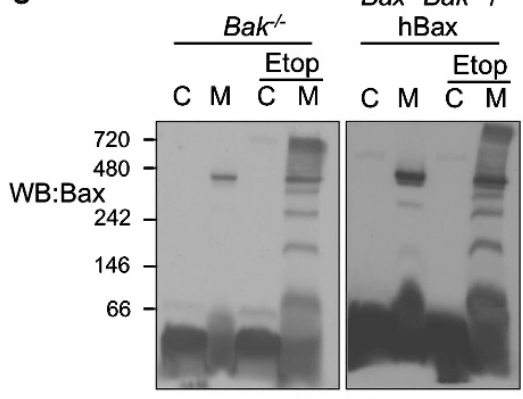

BN-PAGE

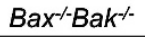

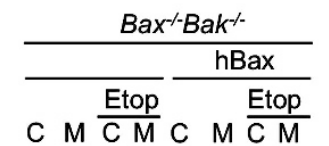

C $M \overline{C M} C M \overline{C M}$

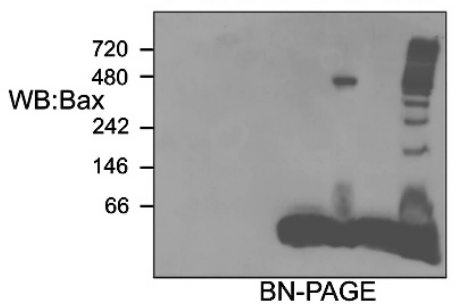

BN-PAGE

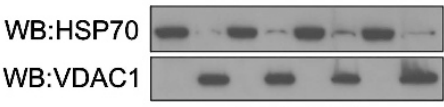

SDS-PAGE

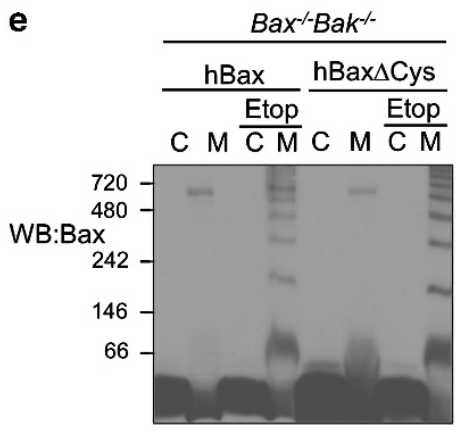

BN-PAGE f

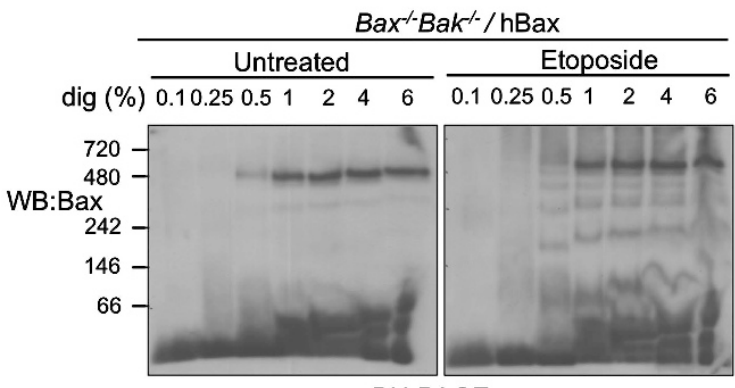

BN-PAGE

Figure 1 Bax forms discrete mitochondrial complexes detectable on BN-PAGE. (a) Bax and Bak exhibit altered subcellular localization in the absence of VDAC2. Wildtype $(\mathrm{wt})$ and $\mathrm{Vdac2}^{-1-}$ MEFs were permeabilized and cytosol $(\mathrm{C})$ and mitochondria-enriched heavy membrane fractions $(\mathrm{M})$ were immunoblotted for Bak, Bax, Bcl- $\mathrm{x}_{\mathrm{L}}$, $\mathrm{Mcl}-1$, cytochrome $c$, VDAC1 (mitochondrial marker) or HSP70 (cytosolic marker). (b) Bax mitochondrial complexes are stable in digitonin. Bak ${ }^{-1-}$ Bax $^{-1-}$ MEFs stably expressing $\mathrm{hBax}$ were treated or not with etoposide in the presence of Q-VD.oph for $24 \mathrm{~h}$. Cytosol $(\mathrm{C})$ and membrane $(\mathrm{M})$ fractions were solubilized in $1 \%$ (w/v) digitonin, Triton X-100 (TX-100) or CHAPS and analysed by BN-PAGE. (c) Endogenous mBax forms similar complexes to ectopically expressed hBax. Bak ${ }^{-1-}$ MEFs or Bak ${ }^{-/-}$Bax $^{-/-}$ MEFs stably expressing hBax were treated or not with etoposide prior to fractionation of cytosol (C) and membrane (M) and analysis by BN-PAGE. (d) Bax complexes detected on BN-PAGE are specific. Cytosol and membrane fractions from Bak ${ }^{-1-} \mathrm{Bax}^{-1-} \mathrm{MEFs}$ or $\mathrm{Bak}^{-1-} \mathrm{Bax}^{-1-}$ MEFs stably expressing hBax were solubilized in $1 \%$ (w/v) digitonin and analysed by BN-PAGE. (e) Bax complexes do not involve disulphide linkage. Cytosol and membrane fractions from Bak ${ }^{-1-} \mathrm{Bax}^{-1-}$ MEFs stably expressing $\mathrm{hBax}$ or hBax in which both endogenous cysteines were mutated to serine (C14S/C166S, hBax $\Delta \mathrm{Cys}$ ) were analysed on BN-PAGE. (f) Bax oligomers are integrated into the MOM, but monomers remain peripheral in apoptotic cells. $\mathrm{Bak}^{-1-} \mathrm{Bax}^{-1-} \mathrm{MEFs}$ were treated or not with etoposide. Membrane fractions were solubilized in the indicated concentration of digitonin (w/v) and analysed by BN-PAGE. All experiments are representative of at least three independent experiments

the higher order Bax homo-oligomers are more stable than Bak homo-oligomers upon removal from the MOM and BN-PAGE. ${ }^{5}$

Bax can associate with VDAC2-deficient mitochondria after an apoptotic stimulus. As our data indicated that Bax as well as Bak interacts with VDAC2 in healthy cells, we investigated the functional relevance of the Bax-VDAC2 interaction with respect to Bax subcellular localization, oligomerization and function. In the absence of VDAC2, Bax localization to the MOM in healthy cells was reduced (Figure 2a, compare lanes 2 and 6). Furthermore, Bax 
a
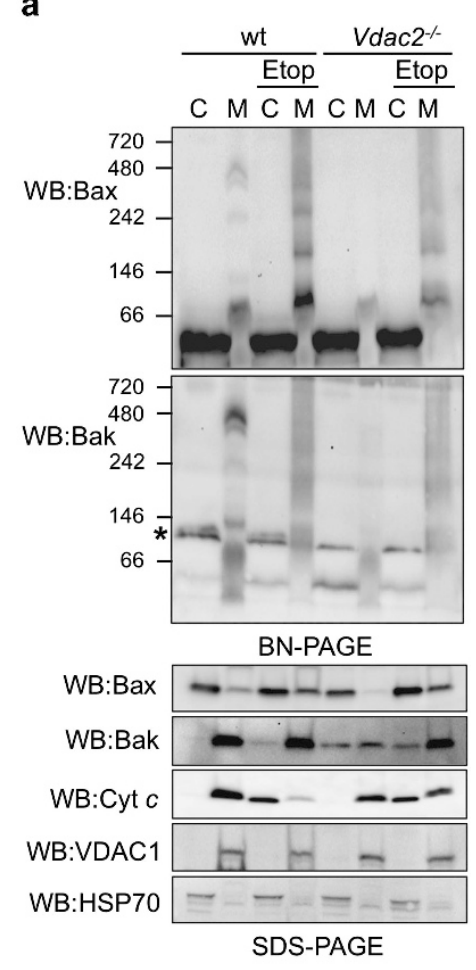

b

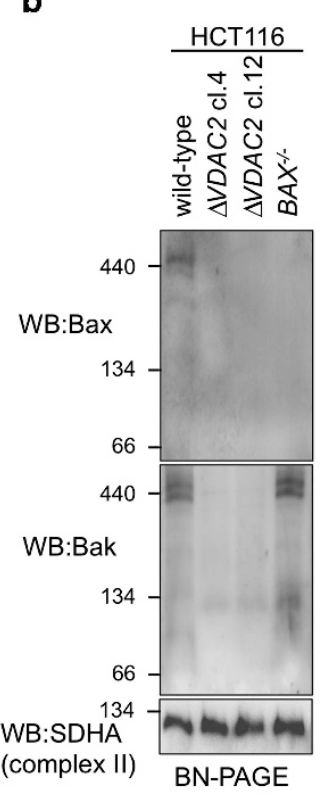

c

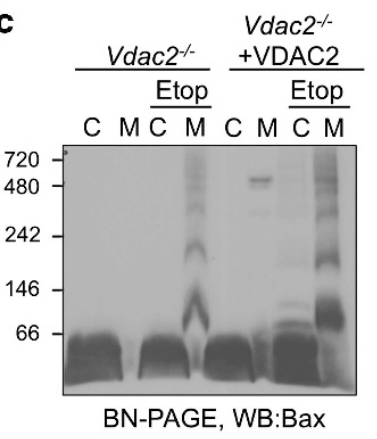

d

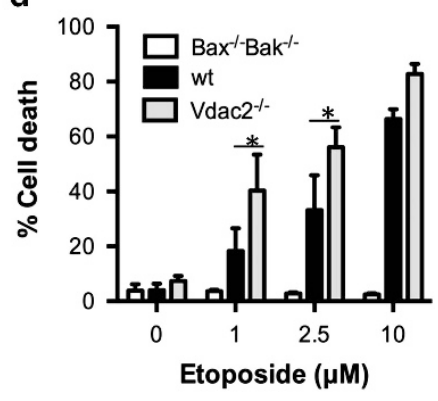

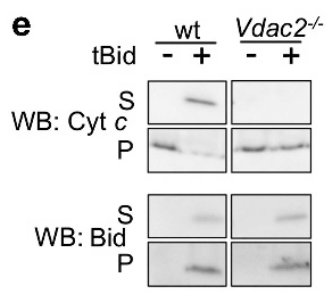

g

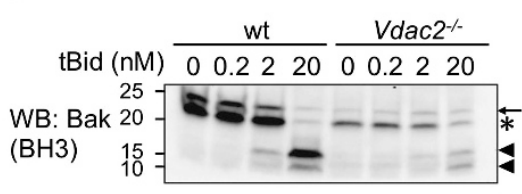

$\mathbf{f}$

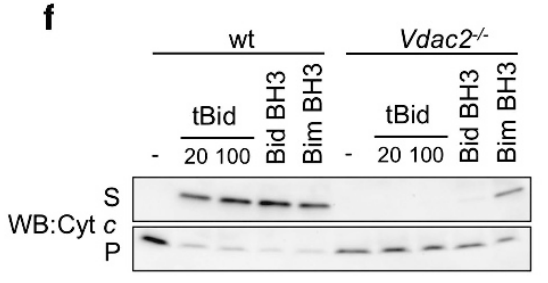

h

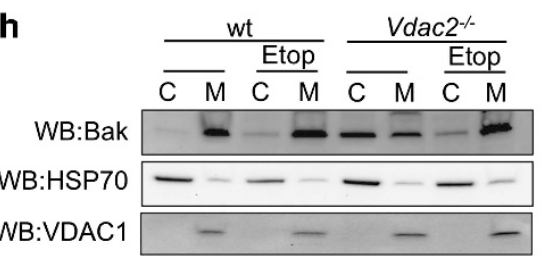

Figure 2 Association with VDAC2 regulates Bax oligomerization. (a) Bax, but not Bak, oligomerization is reduced in cells lacking VDAC2. Wild-type (wt) and Vdac2 ${ }^{-/-}$ MEFs were permeabilized and cytosol (C) and membrane fractions (M) were analysed by BN-PAGE (upper panel) or by SDS-PAGE prior to immunoblotting with the indicated antibodies. * indicates a cross-reactive band detected by the anti-Bak antibody (Supplementary Figure 12). Data are representative of three independent experiments. (b) The large Bax and Bak complex is lost in VDAC2-deficient HCT116 cells. Membrane fractions from $\triangle V D A C 2$ HCT116 cells were assessed by BN-PAGE and immunoblotted for Bax, Bak or SDHA as a loading control. (c) Re-expression of VDAC2 rescues the association of Bax with the $400 \mathrm{kDa}$ complex and enhances Bax oligomerization. Vdac2 ${ }^{-1-}$ MEFs or Vdac2 ${ }^{-1-}$ MEFs stably expressing HA-VDAC2 were treated or not with etoposide prior to analysis of cytosol (C) and membrane fractions (M) by BN-PAGE. Data are

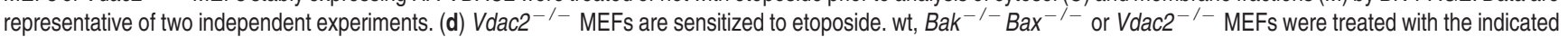
concentration of etoposide for $24 \mathrm{~h}$ prior to analysis of cell death by PI uptake. Note that because of the different genetic background of the Bak ${ }^{-1-} B a x^{-1-}$ cells, direct $^{-1}$ comparison with wt and Vdac2 ${ }^{-1-}$ MEFs is not appropriate, rather the $\mathrm{Bak}^{-/-} \mathrm{Bax}^{-1-}$ are shown to confirm that the cell death induced by etoposide was by apoptosis. Mean \pm S.D. of three independent experiments. * represents $P<0.05$ based on Students unpaired $t$-test. (e) tBid can localize to VDAC2-deficient mitochondria. Membrane fractions from wt or $V d a c 2^{-1-}$ MEFs were treated with recombinant tBid prior to fractionation into supernatant $(\mathrm{S})$ and membrane $(\mathrm{P})$ and immunoblotting. (f) Mitochondrial Bak in Vdac2 $^{-1-}$ MEFs cannot mediate cytochrome $c$ release. Membrane fractions from wt or Vdac2 $^{-/}$MEFs were treated with recombinant tBid at the indicated concentrations (nM) or Bid BH3 or Bim BH3 peptide $(10 \mu \mathrm{M})$ at $30^{\circ} \mathrm{C}$ for 30 min prior to separation of supernatant $(\mathrm{S})$ and pellet $(\mathrm{P})$ and immunoblotting for cytochrome $c$. Data are representative of three independent experiments. (g) Bak in $V_{d a c 2}{ }^{\prime-}$ mitochondria is not hyperactive. Membrane fractions from wt or Vdac2 ${ }^{-/-}$MEFs were treated with the indicated concentrations of recombinant tBid prior to limited proteolysis with proteinase K, SDS-PAGE and immunoblotting with an antibody targeting the Bak BH3 domain. Full-length Bak (arrow) is cleaved in its inactive form $\left(^{*}\right)$ and is further cleaved upon activation (arrowhead). Data are representative of two independent experiments. (h) Bak translocates from the cytosol to mitochondria during apoptosis of $V$ dac2 ${ }^{-1-}$ MEFs. wt or Vdac2 ${ }^{-1-}$ MEFs were treated or not with etoposide prior to fractionation into cytosol (C) and membrane (M) fractions and immunoblotting. Data are representative of four independent experiments 
apoptotic oligomers were reduced in the absence of VDAC2 (Figure 2a).

Bak also exhibited reduced mitochondrial localization in untreated $\mathrm{Vdac2}^{-/-}$MEFs, and was readily detectable in the cytosol (Figure 2a). However, Bak retained an ability to localize to mitochondria and efficiently oligomerize during apoptosis to participate in cytochrome $c$ release (Figure $2 \mathrm{a}$ ). This indicates that VDAC2 facilitates the constitutive targeting of both Bax and Bak to the MOM, but is not absolutely necessary for either Bax or Bak to target mitochondria following an apoptotic stimulus.

Impaired localization of Bak and Bax to $\mathrm{Vdac2}^{-/-}$ mitochondria was not because of a general defect in mitochondrial import or mitochondrial form, as other mitochondrial proteins $\mathrm{Bcl}-\mathrm{x}_{\mathrm{L}}$ and $\mathrm{Mcl}-1, \mathrm{VDAC} 1$ and cytochrome $c$ localized to wild-type and $V d a c 2^{-/-}$mitochondria equally (Figure 1a) and Vdac2 $^{-/-}$cells exhibited no gross defect in mitochondrial morphology (Supplementary Figure 10).

Despite reduced mitochondrial Bak and Bax, $\mathrm{Vdac}^{-/-}$ cells were sensitized to etoposide-induced cell death compared with wild-type MEFs (Figure 2d), although the difference between Vdac2 $^{-/-}$and wild-type MEFs was only statistically significant at lower doses (1 and $2.5 \mu \mathrm{M})$. This heightened response of $\mathrm{Vdac}^{-/-}$cells to apoptotic stimuli is consistent with the proposed role of VDAC2 as a negative regulator of Bak apoptotic function. ${ }^{15}$ To test whether mitochondrial Bak was hyperactive and could efficiently mediate cytochrome $c$ release in the absence of VDAC2 as proposed $^{15}$ or whether recruitment of cytosolic Bak or Bax was necessary, we isolated membrane fractions from wildtype or Vdac2 $^{-/-}$MEFs. Although recombinant tBid could still target $V d a c 2^{-/-}$mitochondria (Figure $2 \mathrm{e}$ ), the resident Bak could not mediate cytochrome $c$ release (Figure $2 e$ ) even in response to high doses of tBid (Figure 2f). Nor could resident Bak mediate cytochrome $c$ release induced by high dose $(10 \mu \mathrm{M})$ of $\mathrm{Bid} \mathrm{BH} 3$ peptide or heat (Figure $2 \mathrm{f}$ and Supplementary Figure 11). Bim $\mathrm{BH} 3$ peptide induced some release from $\mathrm{Vdac}^{-/-}$mitochondria, but still less than with wild-type mitochondria (Figure 2f), likely reflecting the high affinity of Bim for pro-survival proteins. ${ }^{28}$

To test whether Bak in Vdac2 ${ }^{-/}$mitochondria was more prone to undergo conformation change, we performed limited proteolysis. Bak in $\mathrm{Vdac}^{-/-}$MEFs was activated by a similar concentration of tBid $(20 \mathrm{nM})$ as Bak in wild-type mitochondria (Figure $2 \mathrm{~g}$ ). Together our data indicate that the mitochondrial pool of Bak in Vdac2 $2^{-/-}$cells is not hyperactive and is actually insufficient to efficiently mediate MOM permeabilization. Thus, in order for $\mathrm{Vdac}^{-/-} \mathrm{MEFs}$ to die efficiently (Figure 2d), the recruitment of cytosolic Bak (Figures 2a and h) and/or Bax (Figure 2a) is necessary.

Deficiency in both VDAC2 and Bak inhibits Bax mitochondrial association, oligomerization and function during apoptosis. As Bax and Bak can hetero-oligomerize downstream of a death stimulus, ${ }^{5}$ we hypothesized that following an apoptotic stimulus, activated Bak at mitochondria may recruit cytosolic Bax and so bypass the need for VDAC2. Therefore, we tested the effect of silencing Bak expression in Vdac2 $^{-/-}$MEFs on Bax recruitment, oligomerization and function. Immunoblotting confirmed the efficient knockdown of Bax and Bak (Figure 3a). In the absence of VDAC2 and with depleted Bak, Bax oligomerization at the MOM and cytochrome $c$ release was reduced (Figure $3 b$ ). In contrast, in $\mathrm{Vdac}^{-/-}$MEFs with reduced Bax expression, Bak retained an ability to oligomerize at the MOM and mediate cytochrome $c$ release (Figure 3b).

To understand the relative contributions of Bax and Bak to the cell death observed in Vdac2 $2^{-/}$cells, we tested the effect of silencing Bak or Bax expression. Consistent with the phenotypes of $\mathrm{Bak}^{-/-}$and $\mathrm{Bax}^{-/-} \mathrm{MEFs}{ }^{1}$ silencing of neither Bak nor Bax had significant effect on etoposideinduced death in wild-type MEFs (Figure 3c). However, consistent with the failure of Bax to efficiently oligomerize in the MOM in $\mathrm{Vdac}^{-/-} /$shBak MEFs (Figure $3 \mathrm{~b}$ ), these cells exhibited significantly reduced cell death in response to etoposide (Figure 3c). In contrast, Vdac2 $^{-/-}$MEFs with silenced Bax exhibited no reduction in cell death (Figure 3c). Re-expression of VDAC2 was sufficient to rescue the ability of Bax to oligomerize in the MOM (Figure 3d) and cell death in response to etoposide (Figure $3 \mathrm{e}$ ). Thus, the increased death observed in $\mathrm{Vdac2}^{-{ }^{-}}$MEFs is largely attributable to Bak. To test whether the requirement for Bak and/or VDAC2 for Bax apoptotic function was a general phenomenon, we generated $B A K^{-/-}$IVDAC2 HCT116 cells (Supplementary Figure 3). Like MEFs, these double knockout cells exhibited significantly reduced Bax-mediated apoptosis in response to etoposide compared with $B A K^{-/-}$HCT116 cells (Figure 3f). Together, our data indicate that VDAC2 or Bak are necessary in order for Bax to efficiently mediate cell death.

BaxS184L mutant fails to target mitochondria devoid of VDAC2. To test whether VDAC2 plays a role in the formation of the apoptotic pore as well as regulating Bax recruitment to mitochondria, we tested whether targeting Bax to mitochondria by mutagenesis of its C-terminal transmembrane domain was sufficient to bypass the requirement for VDAC2 and Bak and rescue the apoptotic defect in cells lacking both VDAC2 and Bak. Mutagenesis of S184 in the Bax C-terminus to a hydrophobic residue is sufficient to constitutively target Bax to mitochondria. ${ }^{4,29}$ Like wild-type Bax, BaxS184L associated with the large molecular weight VDAC2 complex and oligomerized following an apoptotic stimulus (Figure 4a) as previously observed. ${ }^{30}$ Surprisingly, in contrast to when expressed in wild-type or Bak ${ }^{-1-}$ MEFs, BaxS184L failed to target mitochondria when stably expressed in $\mathrm{Vdac}^{-1-}$ MEFs (Figures $4 \mathrm{~b}$ and $\mathrm{c}$ ). Re-expression of VDAC2 was sufficient to rescue the ability of BaxS184L to constitutively target mitochondria (Figure $4 d$ ). This suggests that the mutation of S184 to a more hydrophobic residue is not sufficient to constitutively target Bax to mitochondria, but rather that the mutation improves the affinity or stability of the association of Bax with VDAC2 resulting in a shift in the equilibrium of Bax from the cytosol to mitochondria. These data further support VDAC2 as an important mediator of Bax mitochondrial localization.

Pro-survival $\mathrm{Bcl}-2$ proteins such as $\mathrm{Bcl}-\mathrm{x}_{\mathrm{L}}$ have also been implicated as important regulators of Bax subcellular localization 
a

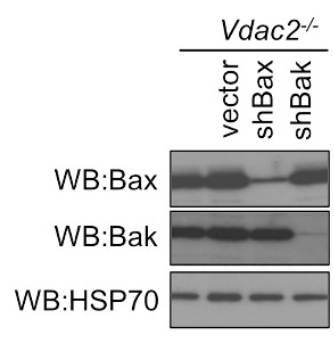

d
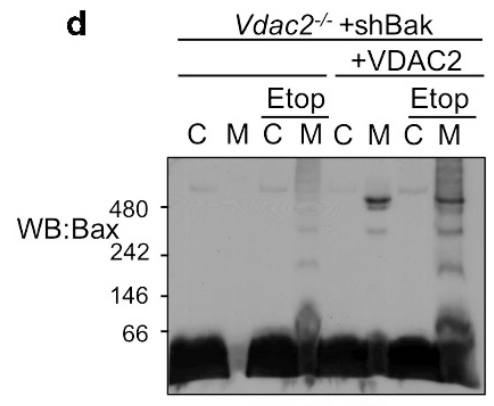

BN-PAGE b

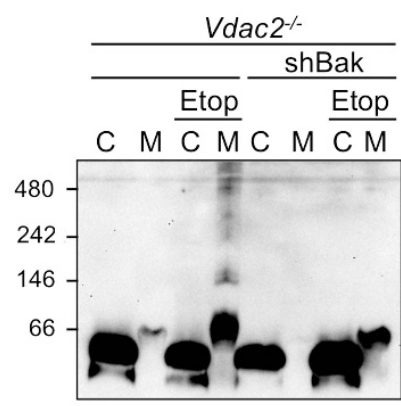

BN-PAGE, WB:Bax

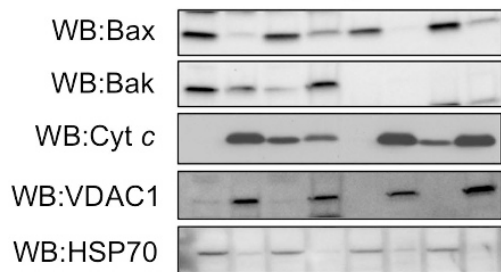

SDS-PAGE

c

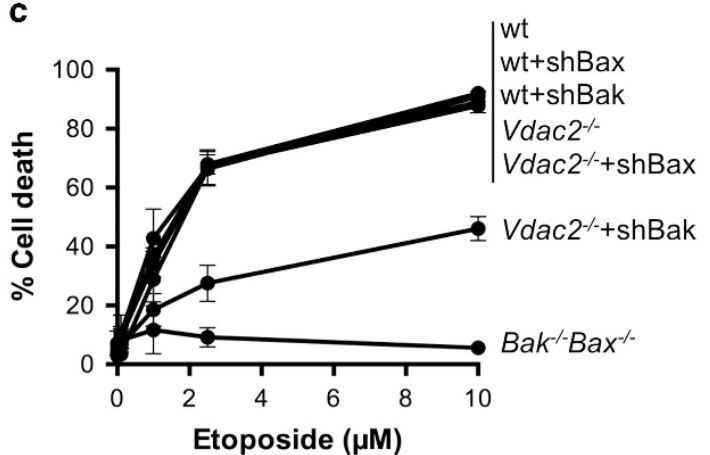

e

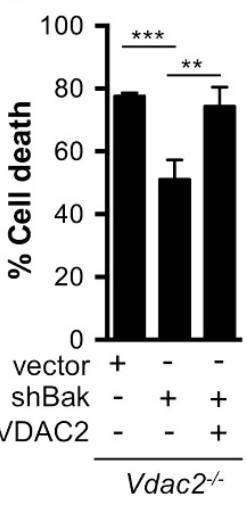

f

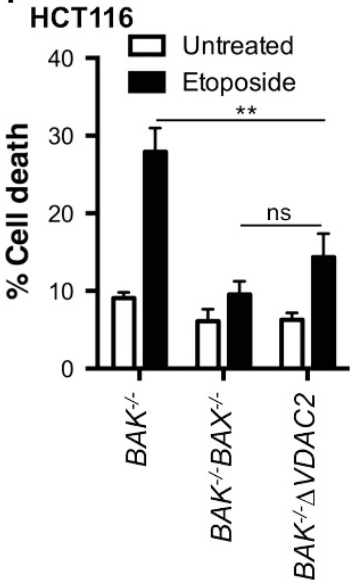

Figure 3 Bax requires either VDAC2 or Bak to efficiently oligomerize in mitochondria and to kill cells. (a) Efficient silencing of endogenous Bak or Bax in Vdac2 ${ }^{-/}-$MEFs. $^{-}$ Vdac2 $^{-1-}$ MEFs were stably infected with empty vector or plasmid containing shRNA targeting mBax or mBak prior to analysis of whole cell lysates by SDS-PAGE and immunoblotting. (b) VDAC2 and Bak are necessary for Bax to efficiently oligomerize at the MOM. Vdac2 ${ }^{-1-}$ MEFs with stable knockdown of Bak or Bax were treated or not with etoposide and cytosol (C) and membrane (M) fractions were analysed by BN-PAGE (upper panel) or SDS-PAGE (lower panels). * indicates a cross-reactive band detected by the anti-Bak antibody. Data are representative of three independent experiments. (c) Cells deficient in VDAC2 and Bak but not VDAC2 and Bax are less sensitive to etoposide. The indicated MEFs lines were treated with the indicated concentration of etoposide for $24 \mathrm{~h}$ prior to analysis of cell death by PI uptake. Mean \pm S.D. of three independent experiments. (d) Re-expression of VDAC2 restores ability of Bax to homo-oligomerize during apoptosis. Vdac2 ${ }^{-/-}$MEFs re-expressing VDAC2 with or without stable knockdown of Bak were treated or not with etoposide and cytosol $(\mathrm{C})$ and membrane $(\mathrm{M})$ fractions were analysed by BN-PAGE. (e) Re-expression of VDAC2 restores apoptotic response to MEFs deficient in VDAC2 and Bak. The indicated MEFs were treated with etoposide $(10 \mu \mathrm{M})$ for $24 \mathrm{~h}$ prior to analysis of cell death by PI uptake. Mean \pm S.D. of four independent experiments. ${ }^{* \star} P=0.001,{ }^{* *} P<0.005$ based on Students unpaired $t$-test. (f) Bax apoptotic function is reduced in the combined absence of Bak and VDAC2. BAK ${ }^{-1-}, B A K^{-1-} B A X^{-1-}$ and $B A K^{-1-} \triangle V D A C 2 \mathrm{HCT} 116$ cells were treated with etoposide $(10 \mu \mathrm{M})$ for $48 \mathrm{~h}$ and assessed for cell death by PI uptake. Data are mean \pm S.D. of three independent experiments. ${ }^{*} P<0.01$ based on Students unpaired $t$-test

by shuttling Bax either towards ${ }^{14}$ or away from ${ }^{13}$ mitochondria. Ectopic expression of $\mathrm{Bcl}-\mathrm{x}_{\mathrm{L}}$ rescued the constitutive ability of BaxS184L to target mitochondria in $\mathrm{Vdac2}^{-1-} \mathrm{MEFs}$ evidenced by confocal microscopy (Figure 4d). This suggests that mitochondrial $\mathrm{Bcl}-\mathrm{x}_{\mathrm{L}}$ allows Bax to accumulate at the MOM rather than actively traffic it to the cytosol. ${ }^{14}$ 
a

$B a k^{-/-B a x}{ }^{-/-}+$BaxS184L

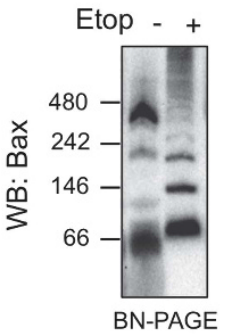

c

GFP-

BaxS184L Mitotracker Merge

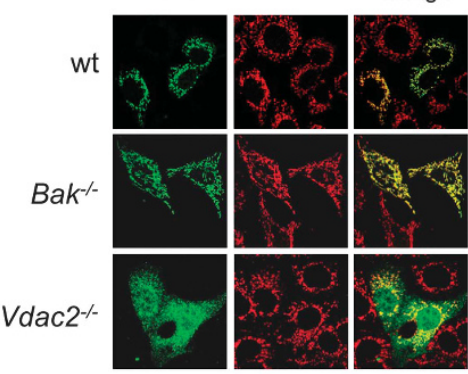

e

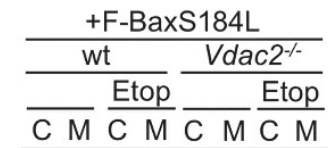

WB:FLAG

WB:HSP70

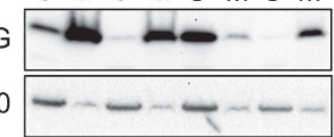

b

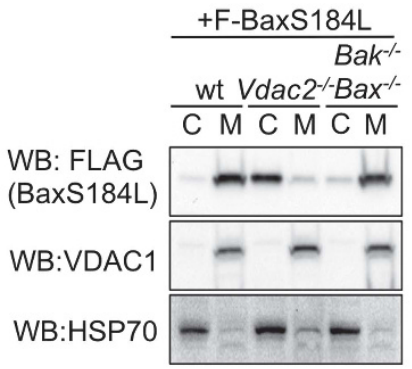

d

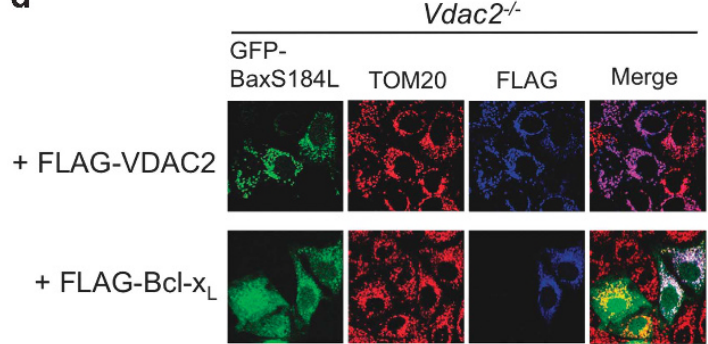

f

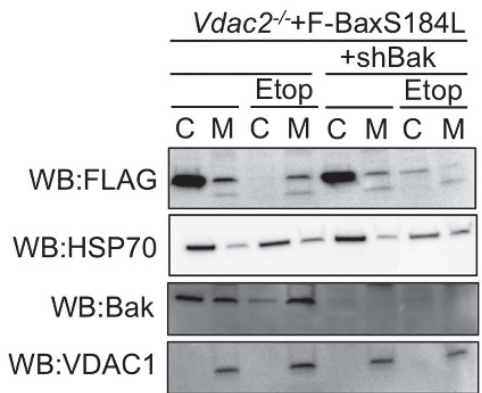

Figure 4 Bax targets mitochondria via distinct mechanisms in healthy and apoptotic cells. (a) BaxS184L associates with VDAC2 in mitochondria. Bak ${ }^{-/-}$Bax ${ }^{-/-}$stably expressing the constitutively mitochondrial Bax mutant S184L were fractionated into cytosol $(\mathrm{C})$ and membrane $(\mathrm{M})$ and analysed by BN-PAGE. Data are representative of three independent experiments. (b) BaxS184L cannot target mitochondria that lack VDAC2. FLAG-BaxS184L was stably expressed in wt, Vdac2 ${ }^{-/-}$or Bak $^{-/-}$Bax $^{-/-}$ MEFs and cytosol (C) and membrane (M) fractions were immunoblotted with the indicated antibodies. Data are representative of three independent experiments. (c) BaxS184L cannot target mitochondria that lack VDAC2. wt, Vdac2 ${ }^{-1-}$ or Bak ${ }^{-1-}$ MEFs were transiently transfected with GFP-BaxS184L, treated with MitoTracker Red CMXRos and analysed by confocal microscopy. (d) Ectopic expression of VDAC2 or Bcl- $x_{L}$ rescues the ability of BaxS184L to target mitochondria. Vdac2 ${ }^{-/-}$MEFs stably expressing FLAG-VDAC2 or FLAG-Bcl- $x_{L}$ were transiently transfected with GFP-BaxS184L prior to immunstaining for TOM20, and FLAG and confocal analysis. (e) BaxS184L can target mitochondria that lack VDAC2 during apoptosis. wt and Vdac2 ${ }^{-1-}$ MEFs stably expressing FLAG-S184L were treated or not with etoposide prior to fractionation into cytosol (C) and membrane (M) fractions and analysis on SDS-PAGE. Data is representative of two independent experiments. (f) BaxS184L requires Bak to target mitochondria during apoptosis. Vdac2 ${ }^{-1-}$ MEFs stably expressing FLAG-S184L with or without stable knockdown of Bak were treated or not with etoposide prior to fractionation into cytosol (C) and membrane (M) fractions and analysis on SDS-PAGE. Data are representative of two independent experiments

To test whether the defect in BaxS184L mitochondrial targeting due to VDAC2-deficiency could be bypassed by Bax activation, we assessed BaxS184L localization in Vdac2 $^{-/-}$ MEFs after an apoptotic stimulus. After etoposide treatment, although overall levels of BaxS184L were reduced, there was a shift of BaxS184L to the membrane fraction (Figure $4 \mathrm{e}$ ), suggesting that activated BaxS184L does not require VDAC2 to target the MOM, consistent with activated Bax-binding Bcl$\mathrm{X}_{\mathrm{L}}$ at mitochondria. ${ }^{31}$ To test whether BaxS184L was recruited by the endogenous mitochondrial pool of Bak in these cells, we silenced Bak expression with shRNA. In the absence of both VDAC2 and Bak, activated BaxS184L failed to target mitochondria following an apoptotic stimulus (Figure 4f).

As the BaxS184L required VDAC2 to target mitochondria, it suggested that the $\mathrm{C}$-terminus of Bax was involved in its interaction with VDAC2, as reported for Bak. ${ }^{20}$ Mutation of a conserved valine in the C-termini of hBak (V198) and hBax (V180) (Figure 5a) impaired association with the VDAC2 complex (Figure 5b, upper panel), but the mutants still targeted to mitochondria (Figure 5b, lower panel). Furthermore, mutation of these $\mathrm{C}$-terminal residues to cysteine also decreased VDAC2 association when these functional mutants were stably expressed in $\mathrm{Bak}^{-/-} \mathrm{Bax}^{-/-}$MEFs (Figure $5 \mathrm{c}$ and d). These data suggest that the Bax and Bak C-termini are important for interaction with VDAC2.

\section{Discussion}

Understanding how Bax and Bak are regulated is paramount for therapeutic targeting of their apoptotic function in disease. Recent evidence has suggested that Bax exists in a dynamic equilibrium between the cytosol and membrane and that 
a

$$
\begin{aligned}
& \text { hsBak. . . GNGPILNVLVVLGVVLLLGQFVVRRFFKS }{ }^{211} \\
& \text { hsBax. . . GTPTWQTVTIFVAGVLLTASLTIWKKMG }
\end{aligned}
$$

b
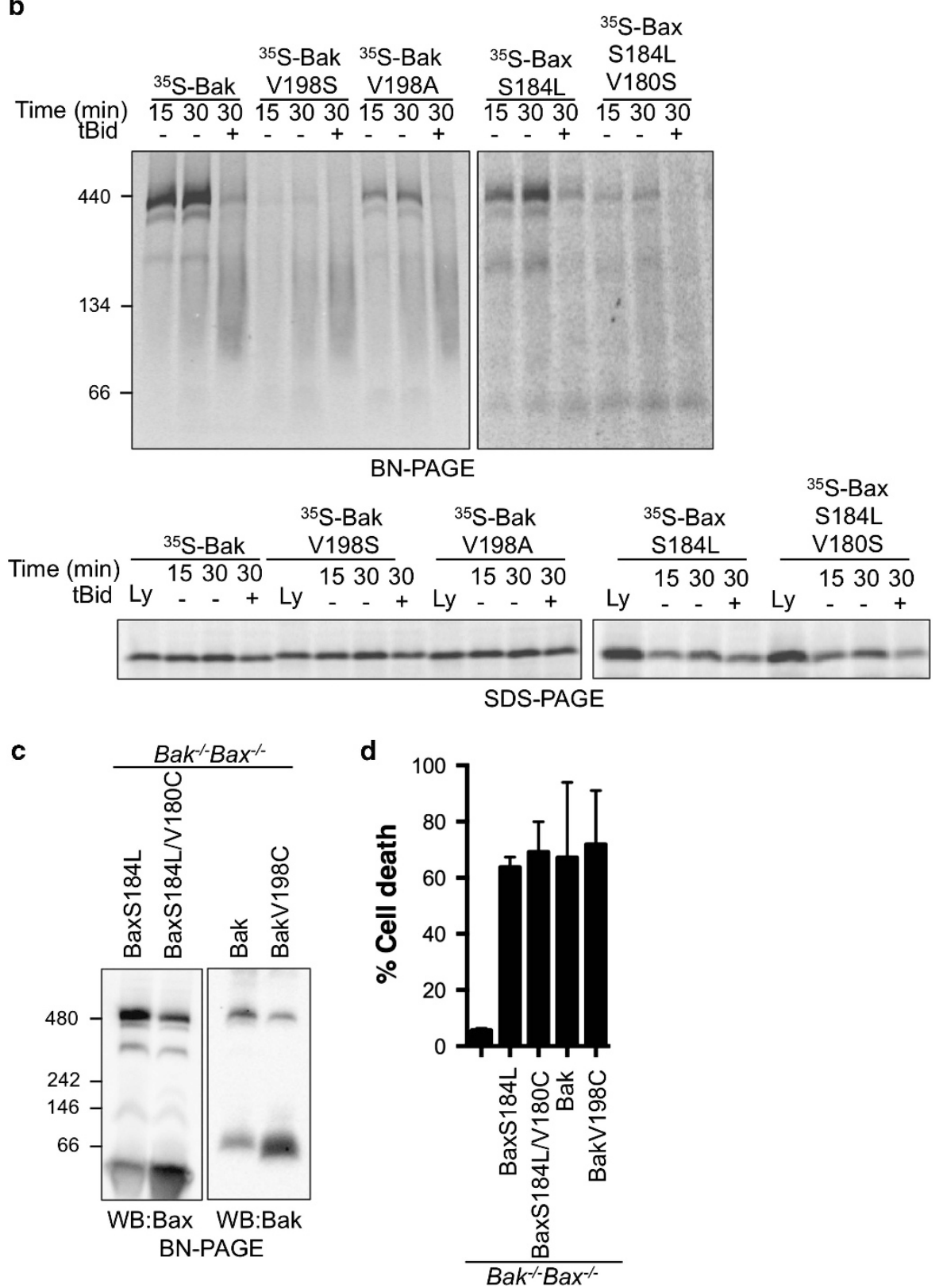

Figure 5 The Bax and Bak C-terminal membrane anchors are important for interaction with VDAC2. (a) Amino acid alignment of human Bak and human Bax. Conserved valine that was mutated to alanine, serine or cysteine is indicated (bold, underlined). (b) Mutation of the Bak and Bax C-termini impairs association with VDAC2 upon import to mitochondria. Mitochondria isolated from wild-type MEFs were incubated with ${ }^{35} \mathrm{~S}$-radiolabelled Bak, BakV198S, BakV198A, BaxS184L or BaxS184L/N180C for the indicated times. Following import, mitochondria were incubated with or without tBid where indicated prior to BN-PAGE (upper panel) or SDS-PAGE (lower panel) and autoradiography. Lysates (Ly) are translated ${ }^{35} \mathrm{~S}$-labelled protein, $20 \%$ input. (c) Mutation of the Bak and Bax C-termini disrupts association with VDAC2 in healthy cells. Membrane fractions from Bak ${ }^{-1-} \mathrm{Bax}^{-1-}$ MEFs stably expressing the indicated Bax or Bak variants were solubilized with $1 \%$ digitonin and analysed by BN-PAGE. (d) Bak and Bax C-termini mutants retained apoptotic function. MEFs described in (c) were treated with etposide (10 mM, $24 \mathrm{~h}$ ) prior to assessment of cell death by PI uptake. Data are mean \pm S.D. of three experiments

altering this equilibrium can alter sensitivity to apoptotic stimuli. ${ }^{13,14}$ However, the molecular mechanisms governing Bax localization and Bax accumulation at the MOM during apoptosis remain controversial. ${ }^{13,14}$ We now propose that Bax employs distinct mechanisms to target mitochondria before and after an apoptotic stimulus. In healthy cells, endogenous Bax that is resident at mitochondria associates with a large molecular weight complex containing VDAC2, as does Bak..$^{20,21}$ 
Whether Bax and VDAC2 interact directly and whether their complex involves additional proteins is unknown. However, Bak is not an intermediary in the Bax-VDAC2 interaction as the complex is observed in $\mathrm{Bak}^{-/-}$cells. $^{5}$

In an earlier study of Bax complexes by BN-PAGE, no discrete Bax complexes were observed either before or after an apoptotic stimulus when membranes were solubilized with CHAPS ${ }^{24}$ Additionally, Bax could co-immunoprecipitate with VDAC1 in neurons solubilized in digitonin, whereas other detergents, including CHAPS, disrupted this interaction. ${ }^{19}$ Our findings are consistent with these studies as supramolecular complexes involving Bax in healthy and apoptotic cells were only resolved when mitochondria were solubilized in digitonin, supporting digitonin as the preferred detergent to examine protein-protein interactions of the Bcl-2 family. 5,19,21 As Bax interacts with VDAC1 in neurons, ${ }^{19}$ it is possible that Bax is regulated by different VDAC isoforms in different cell types. However, because the authors of that study note that the VDAC1 antibody used also immunoreacted with VDAC2 and $\mathrm{VDAC} 3,^{19}$ it is possible that the observed interaction was with VDAC2. This would be consistent with our data showing that high molecular weight complexes involving Bax are undetectable in $\mathrm{Vdac} 2^{-/-}$cells that retain VDAC1 expression. $^{21}$

Our studies support VDAC2 as an important determinant of the constitutive subcellular distribution of Bax and Bak in healthy cells. ${ }^{16,32}$ This role for VDAC2 is seemingly specific for Bak and Bax as the mitochondrial targeting of other $\mathrm{Bcl}-2$ family members including $\mathrm{Bcl}-\mathrm{x}_{\mathrm{L}}, \mathrm{Mcl}-1$ and tBid was not affected by the absence of VDAC2. Recently, Bcl- $x_{L}$ was shown to interact with VDAC $1,{ }^{33}$ but not VDAC2, ${ }^{34}$ suggesting that other VDAC isoforms may play a role in mitochondrial targeting of these Bcl-2 family proteins.

Although constitutive targeting of both Bax and Bak to $V_{d a c 2^{-/-}}$mitochondria was impaired, a population of Bak retained an ability to associate with the MOM. This may implicate a role for alternative mitochondrial receptors for Bak such as VDAC isoforms 1 and 3 or alternatively Bak may integrate spontaneously into the MOM by a process that does not require a membrane receptor. During apoptosis, cytosolic Bax can circumvent VDAC2 to associate with activated mitochondrial Bak and participate in MOM permeabilization. An interaction of activated Bak and Bax involves a BH3groove interaction, ${ }^{5}$ and so it is noteworthy that the mutation of residues in the $\mathrm{Bax} \mathrm{BH} 3$ domain can abrogate mitochondrial targeting. ${ }^{35}$ The ability of $\mathrm{Bcl}-\mathrm{x}_{\mathrm{L}}$ to rescue Bax targeting to mitochondria in the absence of VDAC2 presumably also involves an interaction of the $\mathrm{Bax} \mathrm{BH} 3$ domain with the prosurvival groove. However, the Bax $\mathrm{C}$-terminal tail has also been implicated in its interaction with $\mathrm{Bcl}-\mathrm{x}_{\mathrm{L}}{ }^{36}$

The predominantly cytosolic locale of Bax is facilitated by the sequestration of the C-terminal transmembrane domain in its hydrophobic groove. Substitution of S184 in the Bax C-terminus for a hydrophobic residue disrupts interaction between the tail and groove and constitutively targets Bax to mitochondria. ${ }^{4,29}$ As the mitochondrial localization of BaxS184L was completely abrogated in $\mathrm{Vdac2}^{-/-}$MEFs, our data now show that hydrophobic substitution at $\mathrm{S} 184$ is not sufficient for mitochondrial localization indicating that the mutation does not simply increase the interaction of the transmembrane domain with the lipids of MOM. Rather the mutation likely increases the affinity and/or stability of the association of the Bax C-terminus with VDAC2. BaxS184L associates with mitochondria at a similar rate to wild-type Bax, but its dissociation rate is lower, ${ }^{14}$ again suggesting that altering the tail improves the stability of Bax's association with mitochondria likely mediated by VDAC2. This is consistent with the role for the C-terminal tail of Bak in mediating its interaction with VDAC2, ${ }^{20}$ and the observed preponderance of the large complex with VDAC2 observed with BaxS184L (Figure 4a). Valentijn et al. ${ }^{37}$ have previously reported that the Bax C-terminus is sufficient to target GFP to mitochondria, with the oligomeric state of this fusion protein suggesting that this was probably because of interaction with other mitochondrial proteins. We propose that this mitochondrial protein is VDAC2.

TOM22 has been implicated as a Bax receptor in the MOM. ${ }^{38}$ As mitochondrial import of TOM22 is not impaired in Vdac2 $^{-1-}$ cells, $^{20}$ TOM22 is not sufficient to mediate mitochondrial targeting of Bax in the absence of VDAC2 (and Bak). However, TOM22 and VDAC2 may cooperate in Bax mitochondrial targeting.

Mounting evidence suggests that VDAC2 has an important role in regulating Bak and Bax apoptotic function. Studies have implicated a role for VDAC2 in negatively regulating Bak apoptotic function, ${ }^{15,20}$ with cells lacking VDAC2 sensitized to apoptotic stimuli as Bak was proposed to be hyperactive. ${ }^{15}$ We likewise observed a sensitization to etoposide treatment of $\mathrm{Vdac2}^{-/-}$MEFs compared with wild-type, despite the significant re-distribution of Bak to the cytosol. However, our data indicate that the mitochondrial pool of Bak in $\mathrm{Vdac2}^{-/-}$ MEFs is not in fact hyperactive and is not sufficient to efficiently mediate MOM permeabilization in response to apoptotic stimuli (tBid, Bid BH3, Bim BH3 and heat). Rather, in order for $\mathrm{Vdac2}^{-/-}$cells to die, recruitment of the cytosolic pool of Bak (and Bax) is necessary. That sensitization of Vdac2 $^{-/-}$MEFs was most evident at low doses of etoposide is consistent with previous observations of sensitization only at early time points of etoposide treatment, ${ }^{39}$ and may reflect that etoposide-induced death in wild-type MEFs can be efficiently mediated by Bax. ${ }^{40}$ Thus, any inhibitory effect of VDAC2 on Bak in wild-type MEFs may be obscured at higher doses or later time points.

We propose that downstream of an apoptotic stimulus, cytosolic Bak and Bax in Vdac2 ${ }^{-/-}$MEFs can be recruited to the MOM via interaction with activated mitochondrial Bak, and thus bypass a potentially rate-limiting interaction with VDAC2, to participate in MOM permeabilization and thereby cell death. In contrast, in wild-type MEFs, a significant proportion of Bak and Bax must first dissociate from VDAC2 before it can participate in membrane damage. Thus, our findings reconcile the observation that $\mathrm{Vdac} 2^{-/-}$cells are sensitized to Bakmediated cell death, ${ }^{15,20}$ whilst constitutive recruitment of Bak to the MOM is reduced in the absence of VDAC2. ${ }^{16,39}$

Deletion of Bak rescues the apoptosis defect of $\mathrm{Vdac2}^{-/-}$ thymocytes ${ }^{41}$ supporting a negative regulatory axis for VDAC2 and Bak. ${ }^{15}$ As our data indicate that Bax is unable to efficiently mediate cell death in the absence of both VDAC2 and Bak, one may predict that thymocytes deficient for both VDAC2 and Bak would phenocopy Bak ${ }^{-/-} \mathrm{Bax}^{-/-}$thymocytes. $^{42}$ 
That they do not may indicate that in certain cells, Bax can target mitochondria sufficiently to mediate cell death even in the absence of Bak and VDAC2, perhaps facilitated by interaction with pro-survival $\mathrm{Bcl}-2$ proteins or VDAC $1 .{ }^{13,19}$

In summary, VDAC2 facilitates the mitochondrial association and apoptotic function of Bax. Thus, elucidating the molecular mechanism involved in the Bax-VDAC2 association may reveal a novel target to perturb Bax-mediated apoptosis upstream of mitochondrial damage. Some cell types, such as cerebellar granule neurons, ${ }^{43}$ are reliant upon Bax for their apoptotic response, thus intervention may reveal novel opportunities to protect neuronal cells following stroke.

\begin{abstract}
Materials and Methods
Cell lines, cell culture and induction of apoptosis. MEFs derived from wild-type or $\mathrm{Bak}^{-/-} \mathrm{Bax}^{-/-} \mathrm{C} 57 \mathrm{BL} / 6$ mice, or wild-type or $\mathrm{Vdac2}^{-/-}$ mice on a mixed C57BL/6;129/SvEv background were transformed with SV40 large T and cultured in Dulbecco's Modified Eagles medium supplemented with $10 \%$ foetal calf serum, $250 \mu \mathrm{M} \mathrm{L}$-asparagine and $55 \mu \mathrm{M}$ 2-mercaptoethanol as described. ${ }^{6}$ HeLa and DU145 stably expressing hBax were maintained in Dulbecco's Modified Eagles medium supplemented with 10\% foetal calf serum. Foetal livers were isolated from wt C57BL/6 mice at embryonic day 14.5. Apoptosis was induced in MEFs by treating with etoposide, staurosporine or actinomycin $\mathrm{D}$ for $24 \mathrm{~h}$. Cell death was assessed by flow cytometric analysis of propidium iodide $(\mathrm{PI})$ uptake.
\end{abstract}

Retroviral infection. hBax, hBaxS184L or epitope (HA or FLAG)-tagged VDAC2 in the retroviral expression vector pMX-IRES-GFP (internal ribosome entry site-green fluorescent protein) or short hairpins targeting mBak or mBax in the MSCV LTRmir30 (LMP) vector were retrovirally transduced in MEFs using Phoenix ecotropic packaging cells. ${ }^{6}$ Infected cells were selected on the basis of GFP expression.

Subcellular fractionation and cytochrome $c$ release. MEFs were treated as indicated, harvested and permeabilized in buffer ( $20 \mathrm{mM} \mathrm{Hepes,} \mathrm{pH} 7.5$, $100 \mathrm{mM} \mathrm{KCl}, 2.5 \mathrm{mM} \mathrm{MgCl}, 100 \mathrm{mM}$ sucrose) containing $0.025 \%$ digitonin and supplemented with Complete protease inhibitors without EDTA (Roche, Victoria, Australia). Permeabilization was confirmed by trypan blue uptake and cytosol and mitochondria-enriched heavy membrane fractions were separated by centrifugation at $13000 \mathrm{~g}$ for $5 \mathrm{~min}$ prior to SDS-PAGE analysis. To assess cytochrome $c$ release from isolated mitochondria, pelleted membrane fractions were resuspended in permeabilization buffer without digitonin and incubated for $30 \mathrm{~min}$ with or without caspase-8-cleaved human Bid (tBid), Bid BH3 peptide (SESQEDIIRNIARHLAQVGDSMDRSIPPGLVNGL) at $30^{\circ} \mathrm{C}$ or without stimulus at $43^{\circ} \mathrm{C}$. Supernatant and membrane fractions were separated by centrifugation at $13000 \mathrm{~g}$ for $5 \mathrm{~min}$ prior to SDS-PAGE.

Blue Native-PAGE. BN-PAGE was performed essentially as described. ${ }^{21}$

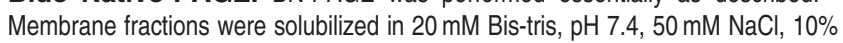
glycerol with $1 \% \mathrm{w} / \mathrm{v}$ (or the indicated concentration) digitonin, $1 \% \mathrm{w} / \mathrm{v}$ CHAPS or $1 \%$ Triton X-100 for $20 \mathrm{~min}$ on ice before centrifugation at $13000 \mathrm{~g}$ to pellet detergent-insoluble debris. BN-PAGE loading dye (5\% Coomassie Blue R-250 (Bio-Rad Laboratories, Gladesville, NSW, Australia) in $500 \mathrm{mM} \mathrm{6-aminohexanoic}$ acid, $100 \mathrm{mM}$ Bis-tris, pH 7.0) was then added to each sample. Gels were electrophoresed in anode buffer ( $50 \mathrm{mM}$ Bis-tris, $\mathrm{pH} 7.0$ ) and blue cathode buffer (50 mM Tricine, $15 \mathrm{mM}$ Bis-tris unbuffered containing 0.02\% Coomassie Blue G-250). Blue cathode buffer was replaced with clear buffer (without Coomassie Blue) when the dye front was $1 / 3$ way through the resolving gel. Gels were transferred to PVDF in Tris-glycine transfer buffer containing 20\% methanol and $0.037 \%$ SDS. Blots were destained in $50 \%$ methanol and $25 \%$ acetic acid and washed with TBS prior to immunoblotting.

Immunoprecipitation. Cells were solubilized on ice for $30 \mathrm{~min}$ in lysis buffer

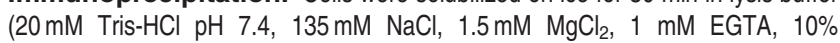
glycerol) containing either $1 \%$ Triton X-100, $1 \%$ digitonin or $1 \%$ CHAPS. Immunoprecipitation was performed as described, ${ }^{6}$ with anti-HA (16B12, Covance, Princeton, NJ, USA). Immunoprecipitates and pre-IP cell lysates were run on
SDS-PAGE under non-reducing or reducing conditions, and immunoblotted for HA (16B12, Covance,) or Bak (aa23-38, Cat. \#B5897, Sigma-Aldrich, Castle Hill, NSW, Australia).

In vitro protein import into isolated mitochondria. Bak, Bcl-2 and $\mathrm{BCl}-\mathrm{X}_{\mathrm{L}}$ were amplified from pGEM4Z using M13 forward and reverse primers to generate RNA using the in vitro mMESSAGE mMachine SP6 kit (Ambion, Life Technologies, Mulgrave, VIC, Australia). RNA was isolated by LiCl precipitation according to the manufacturer's instructions and applied to in vitro translation reactions using rabbit reticulocyte lysate (Promega, Sydney, NSW, Australia) in the presence of $\left[{ }^{35} S\right]$ methionine/cysteine protein labeling mix (PerkinElmer Life Sciences, Melbourne, VIC, Australia). Mitochondria from wild-type MEFs were isolated by differential centrifugation as described previously, ${ }^{44}$ and incubated with translation products in import buffer (20 mM HEPES-KOH, pH 7.4, $250 \mathrm{mM}$ sucrose, $80 \mathrm{mM}$ KOAc, $5 \mathrm{mM}$ MgOAC, $10 \mathrm{mM}$ sodium succinate, $5 \mathrm{mM}$ methionine, $1 \mathrm{mM} \mathrm{DTT}$, and $4 \mathrm{mM} \mathrm{ATP}$ ) at $37^{\circ} \mathrm{C}$ for the indicated times. Membranes were pelleted at $16000 \mathrm{~g}$ for $5 \mathrm{~min}$ at $4^{\circ} \mathrm{C}$ and subsequently solubilized in $1 \%$ digitonin for BN-PAGE or SDS-PAGE loading buffer. Where indicated, mitochondria were incubated in the presence of recombinant tBid $(50 \mathrm{nM})$ in import buffer at $37^{\circ} \mathrm{C}$ for $15 \mathrm{~min}$.

Immunoblotting antibodies. SDS-PAGE gels were immunoblotted for Bak (aa23-38, Cat. \#B5897, Sigma-Aldrich), Bax (N-20, Santa Cruz Biotechnology, Shanghai, China), cytochrome $c$ (clone $7 \mathrm{H} 8.2 \mathrm{C} 12$, BD Biosciences Pharmingen, CA, USA), BCl- $x_{L}$ (\#610212, BD Biosciences, North Ryde, NSW, Australia), Mcl-1 (\#600-401-394, Rockland Immunochemicals, Gilbertsville, PA, USA), HSP70 (from W. Welch, UCSF), VDAC1 (Ab-2, Calbiochem, Billerica, MA, USA), FLAG (M2, Sigma-Aldrich), human VDAC2 (Boris Reljic) or haemagglutinin (HA, 16B12, Covance). Native-PAGE gels were immunoblotted for Bak (7D10, D. C. S. Huang, WEHI) or Bax (21C10, D. C. S. Huang, WEHI), VDAC1 (as above) or SDHA (complex II, Molecular Probes, Mulgrave, VIC, Australia, cat \#A11142). Secondary antibodies were horseradish peroxidase-conjugated anti-rabbit IgG, anti-mouse IgG and anti-rat IgG (Southern Biotech, Birmingham, AL, USA).

TALEN gene targeting. VDAC2 was disrupted in wild-type and $B A K^{-/-}$ HCT116 colorectal cancer cells (a gift from Richard Youle, NIH) using TALEN (transcription activator-like effector nuclease) binding pairs (PMID 22484455) designed to target the common exon of the three isoforms of human VDAC2 as previously described. ${ }^{45}$ Clones were screened for VDAC2-deficiency using an antibody recognizing human VDAC2 (Boris Reljic).

Limited proteolysis. Membrane fractions were incubated with recombinant tBid in the absence of protease inhibitors prior to treatment with proteinase $\mathrm{K}$ $(30 \mu \mathrm{g} / \mathrm{ml})$ for $20 \mathrm{~min}$ on ice. Proteinase $\mathrm{K}$ was quenched with $100 \mathrm{mM}$ PMSF and samples were boiled samples in SDS-PAGE sample buffer. Cleavage fragments were detected by immunoblotting with an internal antibody recognizing the Bak $\mathrm{BH} 3$ domain (4B5). ${ }^{6}$

Confocal analysis. MEFs were seeded overnight on cover slips and then transfected for $20 \mathrm{~h}$ with GFP-BaxS184L with Lipofectamine 2000 (Life Technologies) according to the manufacturer's instructions. Cells were incubated with $50 \mathrm{nM}$ MitoTracker Red CMXRos (Molecular Probes) before being fixed in $4 \%$ (w/v) paraformaldehyde in PBS (pH 7.4) and incubated for $60 \mathrm{~min}$ at room temperature with primary antibody against the FLAG epitope (clone M2, Sigma-Aldrich) or TOM20 (Santa Cruz Biotechnology). Primary antibodies were labeled for $20 \mathrm{~min}$ at room temperature with Alexa Fluor 488-, Alexa Fluor 568-, or Alexa Fluor 647-conjugated anti-rabbit or anti-mouse (Molecular Probes). Confocal microscopy was performed with a Zeiss confocal microscope equipped with a ConfoCor 3 system containing an avalanche photodiode detector. GFP was detected using an argon laser, red fluorescence was detected using a DPSS laser, and deep red fluorescence was detected using a helium/neon laser. All images were processed using ImageJ (http://rsbweb.nih.gov/ij/index.html) and ZENlite 2011 (Blue edition, Zeiss, North Ryde, NSW, Australia).

\section{Conflict of Interest}

The authors declare no conflict of interest. 
Acknowledgements. We would like to thank Stephanie Fennell for technical assistance, Colin Hockings for the Bid and Bim BH3 peptides, Mark van Delft for the FLAG-VDAC2 construct, Gabriela Brumatti for fetal liver extraction, William Craigen for the $V d a c 2^{+/+}$(wild-type) and Vdac2 ${ }^{-/-}$MEFs, Richard Youle for the wildtype, $B A K^{-1-}$ and $B A X^{-1-}$ HCT116 lines and Boris Reljic for the anti-human VDAC2 antibody. The work was supported by grants from the National Health and Medical Research Council of Australia (637335), and the Association for International Cancer Research (10-230), and operational infrastructure grants through the Australian Government IRISS and the Victorian State Government OIS.

1. Wei MC, Zong WX, Cheng EH, Lindsten T, Panoutsakopoulou V, Ross AJ et al. Proapoptotic BAX and BAK: a requisite gateway to mitochondrial dysfunction and death Science 2001; 292: 727-730.

2. Westphal D, Dewson G, Czabotar PE, Kluck RM. Molecular biology of Bax and Bak activation and action. Biochim Biophys Acta 2011; 1813: 521-531.

3. Griffiths GJ, Dubrez L, Morgan CP, Jones NA, Whitehouse J, Corfe BM et al. Cell damageinduced conformational changes of the pro-apoptotic protein Bak in vivo precede the onset of apoptosis. J Cell Biol 1999; 144: 903-914.

4. Nechushtan A, Smith CL, Hsu YT, Youle RJ. Conformation of the Bax C-terminus regulates subcellular location and cell death. EMBO J 1999; 18: 2330-2341.

5. Dewson G, Ma S, Frederick $P$, Hockings $C$, Tan I, Kratina $T$ et al. Bax dimerizes via a symmetric BH3:groove interface during apoptosis. Cell Death Differ 2012; 19: 661-670.

6. Dewson G, Kratina T, Sim HW, Puthalakath H, Adams JM, Colman PM et al. To trigger apoptosis Bak exposes its $\mathrm{BH} 3$ domain and homo-dimerizes via $\mathrm{BH} 3$ :grooove interactions. Mol Cell 2008; 30: 369-380.

7. Korsmeyer SJ, Wei MC, Saito M, Weiler S, Oh KJ, Schlesinger PH. Pro-apoptotic cascade activates $\mathrm{BID}$, which oligomerizes BAK or BAX into pores that result in the release of cytochrome c. Cell Death Differ 2000; 7: 1166-1173.

8. Suzuki M, Youle RJ, Tjandra N. Structure of Bax: coregulation of dimer formation and intracellular localization. Cell 2000; 103: 645-654.

9. Moldoveanu T, Liu Q, Tocili A, Watson M, Shore G, Gehring K. The X-ray structure of a BAK homodimer reveals an inhibitory zinc binding site. Mol Cell 2006; 24: 677-688.

10. Wolter KG, Hsu YT, Smith CL, Nechushtan A, Xi XG, Youle RJ. Movement of Bax from the cytosol to mitochondria during apoptosis. J Cell Biol 1997; 139: 1281-1292.

11. Lindsten T, Ross AJ, King A, Zong W, Rathmell JC, Shiels HA et al. The combined functions of proapoptotic Bcl-2 family members Bak and Bax are essential for normal development of multiple tissues. Mol Cell 2000; 6: 1389-1399.

12. Hsu Y-T, Youle RJ. Bax in murine thymus is a soluble monomeric protein that displays differential detergent-induced conformations. J Biol Chem 1998; 273: 10777-10783.

13. Edlich F, Banerjee S, Suzuki M, Cleland MM, Arnoult D, Wang C et al. Bcl-x $(\mathrm{L})$ Retrotranslocates Bax from the mitochondria into the cytosol. Cell 2011; 145: 104-116.

14. Schellenberg B, Wang P, Keeble JA, Rodriguez-Enriquez R, Walker S, Owens TW et al Bax exists in a dynamic equilibrium between the cytosol and mitochondria to control apoptotic priming. Mol Cell 2013; 49: 959-971.

15. Cheng EH, Sheiko TV, Fisher JK, Craigen WJ, Korsmeyer SJ. VDAC2 inhibits BAK activation and mitochondrial apoptosis. Science 2003; 301: 513-517.

16. Roy SS, Ehrlich AM, Craigen WJ, Hajnoczky G. VDAC2 is required for truncated BIDinduced mitochondrial apoptosis by recruiting BAK to the mitochondria. EMBO Rep 2009; 10: $1341-1347$

17. Shimizu S, Narita M, Tsujimoto Y. Bcl-2 family proteins regulate the release of apoptogenic cytochrome $c$ by the mitochondrial channel VDAC. Nature 1999; 399: 483-487.

18. Tsujimoto Y, Shimizu S. VDAC regulation by the Bcl-2 family of proteins. Cell Death Differ 2000; 7: 1174-1181.

19. Huckabee DB, Jekabsons MB. Identification of Bax-voltage-dependent anion channel 1 complexes in digitonin-solubilized cerebellar granule neurons. J Neurochem 2011; 119 $1137-1150$.

20. Lazarou M, Stojanovski D, Frazier AE, Kotevski A, Dewson G, Craigen WJ et al. Inhibition of Bak activation by VDAC2 is dependent on the Bak transmembrane anchor. J Biol Chem 2010; 285: 36876-36883

21. Ma S, Hockings C, Anwari K, Kratina T, Fennell S, Lazarou M et al. Assembly of the Bak apoptotic pore: A critical role for the Bak alpha6 helix in the multimerization of homodimers during apoptosis. J Biol Chem 2013; 288: 26027-26038.

22. Crichton PG, Harding M, Ruprecht JJ, Lee Y, Kunji ER. Lipid, detergent, and Coomassie Blue G-250 affect the migration of small membrane proteins in blue native gels: mitochondrial carriers migrate as monomers not dimers. J Biol Chem 2013; 288: 22163-22173.
23. Vogel S, Raulf N, Bregenhorn S, Biniossek ML, Maurer U, Czabotar P et al. Cytosolic Bax: does it require binding proteins to keep its pro-apoptotic activity in check? J Biol Chem 2012; 287: 9112-9127.

24. Valentijn AJ, Upton JP, Gilmore AP. Analysis of endogenous Bax complexes during apoptosis using blue native PAGE: implications for Bax activation and oligomerization. Biochem J 2008; 412: 347-357.

25. Annis MG, Soucie EL, Dlugosz PJ, Cruz-Aguado JA, Penn LZ, Leber B et al. Bax forms multispanning monomers that oligomerize to permeabilize membranes during apoptosis. EMBO J 2005; 24: 2096-2103.

26. Oh KJ, Singh P, Lee K, Foss K, Lee S, Park M et al. Conformational changes in BAK, a pore-forming proapoptotic Bcl-2 family member, upon membrane insertion and direct evidence for the existence of $\mathrm{BH} 3-\mathrm{BH} 3$ contact interface in BAK homo-oligomers. J Biol Chem 2010; 285: 28924-28937.

27. Bleicken S, Classen M, Padmavathi PV, Ishikawa T, Zeth K, Steinhoff HJ et al. Molecular details of Bax activation, oligomerization, and membrane insertion. J Biol Chem 2010; 285: 6636-6647.

28. Chen L, Willis SN, Wei A, Smith BJ, Fletcher Jl, Hinds MG et al. Differential targeting of prosurvival $\mathrm{Bcl}-2$ proteins by their $\mathrm{BH} 3-$ only ligands allows complementary apoptotic function. Mol Cell 2005; 17: 393-403.

29. Fletcher Jl, Meusburger S, Hawkins CJ, Riglar DT, Lee EF, Fairlie WD et al. Apoptosis is triggered when prosurvival Bcl-2 proteins cannot restrain Bax. Proc Natl Acad Sci USA 2008; 105: 18081-18087.

30. Ross K, Rudel T, Kozjak-Pavlovic V. TOM-independent complex formation of Bax and Bak in mammalian mitochondria during TNFalpha-induced apoptosis. Cell Death Differ 2009; 16: 697-707.

31. Llambi F, Moldoveanu T, Tait SWG, Bouchier-Hayes L, Temirov J, McCormick LL et al. A unified model of mammalian BCL-2 protein family interactions at the mitochondria. Mol Cell 2011; 44: 517-531.

32. Setoguchi K, Otera H, Mihara K. Cytosolic factor- and TOM-independent import of C-tail-anchored mitochondrial outer membrane proteins. EMBO J 2006; 25: 5635-5647.

33. Arbel N, Ben-Hail D, Shoshan-Barmatz V. Mediation of the antiapoptotic activity of $\mathrm{Bcl}-\mathrm{xL}$ protein upon interaction with VDAC1 protein. J Biol Chem 2012; 287: 23152-23161

34. Huang $\mathrm{H}, \mathrm{Hu} \mathrm{X}$, Eno $\mathrm{CO}$, Zhao G, Li C, White C. An interaction between Bcl-xL and the voltage-dependent anion channel (VDAC) promotes mitochondrial $\mathrm{Ca} 2+$ uptake. J Biol Chem 2013; 288: 19870-19881.

35. Kim H, Tu HC, Ren D, Takeuchi O, Jeffers JR, Zambetti GP et al. Stepwise activation of BAX and BAK by tBID, BIM, and PUMA initiates mitochondrial apoptosis. Mol Cell 2009; 36: $487-499$

36. Todt F, Cakir Z, Reichenbach F, Youle RJ, Edlich F. The C-terminal helix of Bcl-x(L) mediates Bax retrotranslocation from the mitochondria. Cell Death Differ 2013; 20: 333-342.

37. Valentijn AJ, Upton JP, Bates N, Gilmore AP. Bax targeting to mitochondria occurs via both tail anchor-dependent and -independent mechanisms. Cell Death Differ 2008; 15: 1243-1254

38. Bellot G, Cartron PF, Er E, Oliver L, Juin P, Armstrong LC et al. TOM22, a core component of the mitochondria outer membrane protein translocation pore, is a mitochondrial receptor for the proapoptotic protein Bax. Cell Death Differ 2007; 14: 785-794.

39. Yamagata H, Shimizu S, Nishida Y, Watanabe Y, Craigen WJ, Tsujimoto Y. Requirement of voltage-dependent anion channel 2 for pro-apoptotic activity of Bax. Oncogene 2009; 28 : 3563-3572.

40. Willis SN, Chen L, DEWSON G, Wei A, Naik E, Fletcher Jl et al. Pro-apoptotic Bak is sequestered by $\mathrm{Mc1}-1$ and $\mathrm{Bcl}-\mathrm{xL}$, but not $\mathrm{Bcl}-2$, until displaced by $\mathrm{BH}$-only proteins. Genes Dev 2005; 19: 1294-1305.

41. Ren D, Kim H, Tu HC, Westergard TD, Fisher JK, Rubens JA et al. The VDAC2-BAK rheostat controls thymocyte survival. Sci Signal 2009; 2: ra48.

42. Rathmell JC, Lindsten T, Zong W-X, Cinalli RM, Thompson CB. Deficiency in Bak and Bax perturbs thymic selection and lymphoid homeostasis. Nat Immunol 2002; 3: 932-939.

43. Putcha GV, Deshmukh M, Johnson EM Jr. Inhibition of apoptotic signaling cascades causes loss of trophic factor dependence during neuronal maturation. J Cell Biol 2000; 149: 1011-1018.

44. Lazarou M, McKenzie M, Ohtake A, Thorburn DR, Ryan MT. Analysis of the assembly profiles for mitochondrial- and nuclear-DNA-encoded subunits into complex I. Mol Cell Biol 2007; 27: 4228-4237.

45. Stroud DA, Formosa LE, Wijeyeratne XW, Nguyen TN, Ryan MT. Gene knockout using transcription activator-like effector nucleases (TALENs) reveals that human NDUFA9 protein is essential for stabilizing the junction between membrane and matrix arms of complex I. J Biol Chem 2013; 288: 1685-1690. 